\title{
Programa mínimo para análises de ecossistemas: \\ Artrópodos terrestres em florestas inundáveis da Amazônia Central (*)
}

\author{
Joachim Adis ("म)
}

\begin{abstract}
Resumo
No campo da regiẫo de águas pretas (rio Ta rumā-mirim) e no campo da região de águas brancas (rio Solimōes), foram instalados desde janeiro de 1976: 28 armadilhas de solo, 4 fotoeclectores do solo e 13 fotoeclectores de árvore (Programa Mínimo para Análise de Ecossistemas: Grimm, Funke \& Schauermann, 1974). Nos fotoeclectores das árvores, capturamos até maio de 1976 no campo do Solimôes 18.197 artrópodos e sapos. Foram dominantes as formigas (14.068 individuos $=77,31 \%$ ) e dipteros ( 1.634 indivíduos $=8,98 \%$ ). No campo do Tarumā-mirim capturamos durante o mesmo tempo 27.594 artrópodos e gastrópodos. Foram dominantes os pseudoescorpiōes $(7.942$ indivíduos $=$ $28,78 \%$ ), pequenas aranhas $(5.878$ indivíduos $=$ $21,30 \%)$ e formigas $(5.422$ indivíduos $=19,65 \%)$. No campo do Soïimōes falta a fauna do solo (porque não tem "litter") e só as grandes aranhas fazem migraçōes antes das enchentes. No campo do rio Tarumä-mirim temos 8 grupos de insetos que fazem migrações, principalmente a fauna do solo. As migraçōes feitas pelos insetos começam de 6 a 8 semanas ou na última semana antes das enchentes do campo.
\end{abstract}

\section{INTRODUÇÃo}

Os níveis da água do rio Solimões e do rio Negro variam durante o ano. Em Manaus a diferença entre o nível de enchente e o de vazante atinge até 15 metros. Durante a enchente, extensas regiões florestadas às margens dos rios são inundadas. A adaptação dos animais e a importância dos Artrópodos terrestres, no ecossistema "floresta inundável", são ainda Insufıcientemente conhecidas (Beck, 1971; Schaller, 1973) .

Migrações horizontais e verticais que são dependentes de flutuações periódicas do nívei de água foram estudadas apenas preliminarmente (Beck, 1968, 1972; Beck \& Schubart, 1968; Irmler, 1973, 1974).
As capturas com fotoeclectores de árvores (veja Figura 1) durante a enchente, permitiram as primeiras comparações sobre a fauna de Artrópodos terrestres de uma floresta inundável em água preta e em água branca.

\section{ÁREA DE ESTUdo}

A floresta de inundação na região de água preta (= igapó; compare Sioli, 1956) está situada no curso inferior do rio Tarumã-mirim. próximo a confluência com o rio Negro, aproxi.

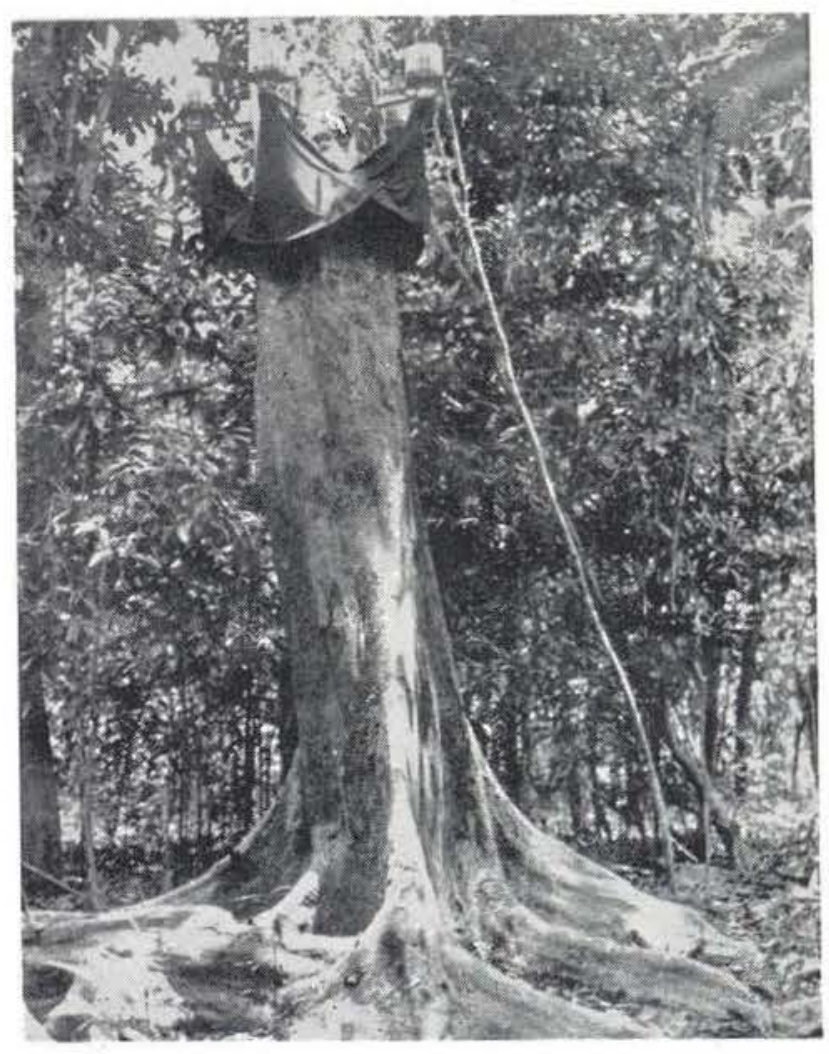

Fig. 1 - Fotoeclector de árvore na área experimental do rio Tarumă-mirim.


(Studienstiftung des Deutschen Volkes).

(*) - II. Zoologisches Institut der Universität Göttingen, Abtlg. Ökologie, Göttingen, Alemanha Oc. / Instituto Nacional de Pesquisas da Amazônia (INPA), Manaus, Brasil. 
madamente a $20 \mathrm{~km}$ acima de Manaus. O solo rico em raízes está coberto por uma densa camada de folhas de $5-10 \mathrm{~cm}$ de espessura.

A floresta inundável na região de água branca (= várzea; compare Sioli, 1956) está situada na ilha do Curari, na margem direita do rio Solimões a cerca de $15 \mathrm{~km}$ acima da confluência do rio Negro com o Amazonas. Sobre o solo pobre em raízes (predominantemente sedimentação de substâncias inorgânicas em suspensão constituídas de quartzo, feldspatos e minerais argilosos) encontra-se uma camada de folhas, pouco espessa, aberta.

Uma descrição mais completa da topografia, geomorfologia e vegetação das áreas de estudo encontram-se em Irmler (1974).

\section{MÉTodos}

Populações de Artrópodos terrestres, em Ecossistemas Florestais, podem ser inventariados por meio do uso combinado de três aparelhos de captura: 0 fotoeclector de solo (Funke, 1971), a armadilha de solo (Adis, 1974) e o fotoeclector de árvores (Funke, 1971). Este "programa mínimo para análise de ecossistemas" (Grimm Funke \& Schauermann, 1974) - desenvolvido durante o projeto I.B.P.Solling na Alemanha (Ellenberg, 1971) - permite afirmações sobre inventário de espécies, estrutura de dominância, fenologia e abundância de Artrópodos.

Em dezembro de 1975 foram instalados, nas duas áreas experimentais, um total de 26 fotoeclectores de árvores. Em cada área havia duas árvores com quatro fotoeclectores, e uma árvore com cinco fotoeclectores a uma altura de $4 \mathrm{~m}$. As árvores escolhidas (distantes $10 \mathrm{~m}$ até $20 \mathrm{~m}$ ) encontravam-se no local mais alto da área experimental (compare Irmler, 1974: 今 área 3)

Durante a fase seca (até 22.3.76) foram colocados mais quatro fotoeclectores de solo e 28 armadilhas de solo em cada área.

Foi usado como líquido de captura, uma soluçâo de ácido pícrico sem detergente (uma parte de solução de ácido pícrico saturado e três partes d'água). Durante experimentos no campo e no laboratório esta solução mostrouse quase sempre neutra para captura (Adis 1974, 1975). Semanalmente o liquido dos aparelhos foi trocado e o material coletado.

Até 22 de março 1976, as duas áreas experimentais ficaram secas. Durante a última semana de março com a enchente, a água chegou na base das árvores com os fotoeclectores (inundação total da área), e no final de maio na borda interior do funil dos fotoeclectores de árvores.

Até agora foram avaliadas as capturas dos fotoeclectores de árvores (janeiro até maio 1976) das duas áreas experimentais. Os dados obtidos permitem afirmaçc̃es comparativas sobre o inventário de espécies, a estrutura de dominância, a ascensão no tronco, a densidade de população na área do tronco e sobre os tipos de migração durante a enchente.

Até agora não foram ainda avaliados: Proturas, Colêmbolas, Tisanópteros, larvas de Coleópteros, Ácaros e larvas de Dípteros.

\section{RESUltados}

\section{INVENTÁRIO DE ESPÉCIES / ESTRUTURA} DE DOMINÂNCIA

$\mathrm{Na}$ área experimental do rio Solimões foram capturados de janeiro até maio 1976 (região de água branca) um total de 18.197 Artrópodos e Sapos nos fotoeclectores de árvores (veja tabela I).

São dominantes: Formigas (14.068 Ind. = $77,31 \%$ ) e Dípteros (1.634 Ind. $=8,98 \%$ ).

São menos freqüentes: Coleópteros, Aranhas grandes, pequenas Saltatórias e larvas de Blatídeos $(1,10-3,60 \%)$.

Os 13 grupos restantes aparecem esporadicamente $(0,06-0,47 \%)$.

$\mathrm{Na}$ área experimental do rio Tarumã-mirim (região de água preta) foram capturados no mesmo período um total de 27.594 Artrópodos e Gastrópodos (veja tabela I).

Três grupos são dominantes: Pseudoescorpiões $(7.942$ Ind. $=28,78 \%)$, pequenas Aranhas $(5.878$ Ind. $=21,30 \%)$ e Formigas $(5.422$ Ind. $=19,65 \%$ ). Nove grupos são menos fre- 
TABELA I (") - Capturas com fotoeclectores de árvore: "estrutura de dominância" (janeiro-maio 1976); $\mathbf{N}=\mathbf{n} \cdot{ }^{\circ}$ de individuos.

\begin{tabular}{|c|c|c|c|c|c|c|c|c|}
\hline & Solimões & & $\mathbf{N}$ & $\%$ & & Tarumã-mirim & $\mathbf{N}$ & $\%$ \\
\hline 1 & Formicoidea & & 14.068 & 77,31 & 1 & Pseudoscorp. & 7.942 & 28,78 \\
\hline 2 & Diptera & & 1.634 & 8,98 & 2 & Araneae peq. & 5.878 & 21,30 \\
\hline 3 & Coleoptera & & 655 & 3,60 & 3 & Formicondea & 5.422 & 19,65 \\
\hline 4 & Araneae peq. & & 608 & 3,34 & 4 & Isopoda & 1.497 & 5,43 \\
\hline 5 & Saltatoria & & 385 & 2,12 & 5 & Homoptera lv. & 1.032 & 3,74 \\
\hline 6 & Araneae grd. & & 239 & 1,31 & 6 & Chilopoda & 967 & 3,50 \\
\hline 7 & Blattidae lv. & & 200 & 1,10 & 7 & Diptern & 929 & 3,37 \\
\hline 8 & Homoptera lv. & & 85 & 0,47 & 8 & Symphyla & 877 & 3,18 \\
\hline 9 & Hymenoptera & & 71 & 0,39 & 9 & Diplopoda & 663 & 2,40 \\
\hline 10 & Mantidae & & 50 & 0,27 & 10 & Araneae grd. & 479 & 1,74 \\
\hline 11 & Hemiptera & & 38 & 0,21 & 11 & Blattidee lv. & 417 & 1,51 \\
\hline 12 & Lepidoptera ad. & & 32 & 0,18 & 12 & Saltatoria & 331 & 1,20 \\
\hline \multirow[t]{2}{*}{13} & Blattidae ad. & & 15 & 0,08 & 13 & Coleoptera & 219 & 0,79 \\
\hline & [Ranidae & & & & 14 & Psocoptera & 146 & 0,53 \\
\hline 14 & Pseudoscorp. & & 14 & 0,08 & 15 & Hymenoptera & 115 & 0,42 \\
\hline \multirow[t]{2}{*}{15} & Apidae & & 13 & 0,07 & 16 & Apidae & 100 & 0,36 \\
\hline & [Lepidoptera lv. & & & & 17 & Mantidae & 97 & 0,35 \\
\hline \multirow[t]{2}{*}{16} & Psocoptera & & 12 & 0,06 & 18 & Machilidae & 93 & 0,34 \\
\hline & [ Dermaptera & & & & 19 & Hemiptera & 66 & 0,24 \\
\hline \multirow[t]{2}{*}{17} & Homoptera ad. & & 11 & 0,06 & 20 & Uropygi & 65 & 0,24 \\
\hline & outros & & & & 21 & Lepidoptera lv. & 36 & 0,13 \\
\hline \multirow{8}{*}{18} & outros & & 67 & 0,37 & 22 & Homoptera ad. & 33 & 0,12 \\
\hline & total & & $\overline{18.197}$ & - & 23 & Embioptera & 26 & 0,09 \\
\hline & & & 18.197 & 100,00 & 24 & Opiliones & 22 & 0,08 \\
\hline & & & & & 25 & Lepidoptera ad. & 19 & 0,07 \\
\hline & & & & & 26 & Blattidae ad. & 18 & 0,06 \\
\hline & & & & & 27 & Gastropoda & 10 & 0,04 \\
\hline & & & & & 28 & outros & 95 & 0,34 \\
\hline & & & & & & total & 27.594 & 100,00 \\
\hline
\end{tabular}

(*) - Dados preliminares.

qüentes: Isópodos, larvas de Homópteros, Quilópodos, Dípteros, Sínfilos, Diplópodos, Aranhas grandes, larvas de Blatídeos e Saltatórias $(1,20-5,43 \%)$.

Os 15 grupos restantes aparecem esporadicamente $(0,04-0,79 \%)$.

Em total foram encontrados na região de água preta 26 grupos de Artrópodos e na região de água branca somente 19 grupos.

$\mathrm{Na}$ área experimental do rio Solimões a fauna típica do solo, quase não existe (veja tabela II). As Aranhas pequenas, que já foram capturadas deverão ser examinadas se são es. pécies que habitam as árvores ou não.

$\mathrm{Na}$ região de água branca não foram capturados Sínfilos, Isópodos, Maquilídeos, Urópigos e Embiópteros.
TABELA II (*) - N.० de artrópodos capturados por meio de fotoeclectores de árvore: "fauna do solo" (janeiro-maio 1976).

Tarumã-mirim Rio Solimões

$\begin{array}{lrr}\text { Pseudoscorpionida } & 7942 & 14 \\ \text { Araneae peq. } & 5878 & (608) \\ \text { Isopoda } & 1497 & / \\ \text { Chilopoda } & 967 & 3 \\ \text { Symphyla } & 877 & / \\ \text { Diplopoda } & 663 & 12 \\ \text { Machilidae } & 93 & / \\ \text { Uropygi } & 65 & / \\ \text { Opiliones } & 22 & 1\end{array}$

(*) - Dados preliminares. 
Concluindo os resultados até o momento, na região de água preta, a biomassa, o número de indivíduos e espécies, parece ser maior do que na região de água branca, o que contraria a hipótese de Janzen (1974), segundo a qual seria de se esperar uma comunidade reduzida de insetos nas regiões de água preta devido à altá taxa de toxinas nas folhas das plantas nesta região, que então não poderiam ser consumidas pelos animais.

ASCENSÃO NO TRONCO / DENSIDADE DE POPULAÇ̃̃o NA ÁREA DO TRONCO

No início da enchente na área experimental do Tarumã-mirim, foi constatada uma evasão, para as árvores, de oito grupos de Artrópodos, principalmente os habitantes do solo

TABELA III (") - Capturas com fotoeclectores de árvore: "n. de indivíduos por mês (janeiro-maio 1976) - Rio Solimões -"

\begin{tabular}{|c|c|c|c|c|c|c|}
\hline & & $\mathbf{J}$ & $\mathbf{F}$ & M & $\mathbf{A}$ & M \\
\hline 1 & Formicoidea & 6805 & 4743 & 1926 & 419 & 175 \\
\hline 2 & Diptera & 463 & 275 & 506 & 176 & 214 \\
\hline 3 & Coleoptera & 258 & 227 & 34 & 32 & 104 \\
\hline 4 & Araneae peq. & 154 & 106 & 108 & 48 & 192 \\
\hline 5 & Saltatoria & 100 & 86 & 136 & 31 & 32 \\
\hline 6 & Araneae grd. & 46 & 45 & 109 & 21 & 18 \\
\hline 7 & Blattidae lv. & 96 & 73 & 17 & 9 & 5 \\
\hline 8 & Homoptera lv. & 49 & 28 & 4 & 3 & 1 \\
\hline 9 & Hymenoptera & 25 & 25 & 13 & 1 & 8 \\
\hline 10 & Mantidae & 41 & 5 & 2 & I & 2 \\
\hline 11 & Hemiptera & 24 & 9 & 4 & I & 1 \\
\hline 12 & Lepidoptera ad. & 6 & 1 & 1 & I & 24 \\
\hline 13 & Blattidae ad. & 11 & 4 & I & 1 & l \\
\hline 14 & Ranidae & 3 & I & 2 & 4 & 5 \\
\hline 15 & Pseudoscorp. & 4 & 5 & 3 & 1 & 1 \\
\hline 16 & Apidae & 12 & l & l & 1 & 1 \\
\hline 17 & Lepidoptera 1v. & 4 & 5 & 1 & 1 & 1 \\
\hline 18 & Psocoptera & 4 & 4 & 4 & 1 & I \\
\hline 19 & Dermaptera & 1 & 1 & 1 & 1 & 9 \\
\hline 20 & Homoptera ad. & 5 & 2 & I & 1 & 3 \\
\hline (21) & Staphylinidae & 3 & 1 & 5 & 7 & 59 \\
\hline (22) & Cerambycidae & 4 & 18 & 3 & 4 & 1 \\
\hline (23) & Curculionidae & 7 & 5 & 2 & 2 & 1 \\
\hline
\end{tabular}

(*) - Dados preliminares. (veja tabela V A, tabela IV). Dos sete grupos, principalmente os voadores, a densidade de população na área do tronco foi estável até o fim de março, diminuindo muito depois (veja tabela V C, tabela IV).

Parece que Coleópteros e larvas de Lepidópteros surgem mais freqüentemente durante o período de seca (veja tabela V B, tabela IV).

$\mathrm{Na}$ área experimental do rio Solimões só as Aranhas grandes migram para as árvores (veja tabela V A, tabela II).

Parece que existe uma maior densidade de população na área do tronco durante o período de seca (veja tabela V B, tabela III).

TABELA IV (") - Capturas com fotoeclectores de árvore: "n.॰ de indivíduos por mês (janeiro-maio 1976) - Rio Tarumã-mirim -”

\begin{tabular}{|c|c|c|c|c|c|c|}
\hline & & $\mathbf{J}$ & $\mathbf{F}$ & M & $\mathbf{A}$ & M \\
\hline 1 & Pseudoscorp. & 196 & 1810 & 5087 & 253 & 596 \\
\hline 2 & Araneae peq. & 137 & 223 & 4800 & 402 & 316 \\
\hline 3 & Formicoidea & 1779 & 1523 & 1869 & 155 & 96 \\
\hline 4 & Isopoda & I & 1 & 1402 & 91 & 3 \\
\hline 5 & Homoptera 1v. & 233 & 271 & 496 & 22 & 10 \\
\hline 6 & Chilopoda & 73 & 93 & 654 & 47 & 100 \\
\hline 7 & Diptera & 325 & 187 & 218 & 97 & 102 \\
\hline 8 & Symphyla & I & 148 & 686 & 28 & 15 \\
\hline 9 & Diplopoda & 129 & 71 & 194 & 222 & 47 \\
\hline 10 & Araneae grd. & 67 & 104 & 244 & 29 & 35 \\
\hline 11 & Blattidae lv. & 68 & 60 & 250 & 28 & 11 \\
\hline 12 & Saltatoria & 124 & 86 & 102 & 14 & 5 \\
\hline 13 & Coleoptera & 95 & 47 & 49 & 14 & 14 \\
\hline 14 & Psocoptera & 54 & 22 & 21 & 35 & 14 \\
\hline 15 & Hymenoptera & 41 & 17 & 50 & 4 & 3 \\
\hline 16 & Apidae & 30 & 25 & 45 & l & 1 \\
\hline 17 & Mantidae & 30 & 15 & 42 & 8 & 2 \\
\hline 18 & Machilidae & 25 & 33 & 35 & I & I \\
\hline 19 & Hemiptera & 17 & 11 & 30 & 4 & 4 \\
\hline 20 & Uropygi & 12 & 6 & 47 & I & 1 \\
\hline 21 & Lepidoptera Iv. & 21 & 8 & 6 & 1 & 1 \\
\hline 22 & Homoptera ad. & 10 & 8 & 8 & 2 & 5 \\
\hline 23 & Embioptera & 4 & 5 & 12 & 3 & 2 \\
\hline 24 & Opiliones & 3 & 1 & 5 & 13 & , \\
\hline 25 & Lepidoptera ad. & 1 & 6 & 8 & / & 5 \\
\hline 26 & Blattidae ad & 4 & 3 & 9 & 2 & 1 \\
\hline 27 & Gastropoda & 2 & 1 & 6 & 1 & I \\
\hline (28) & Curculionidae & 62 & I & 1 & I & I \\
\hline
\end{tabular}

(*) - Dados preliminares. 

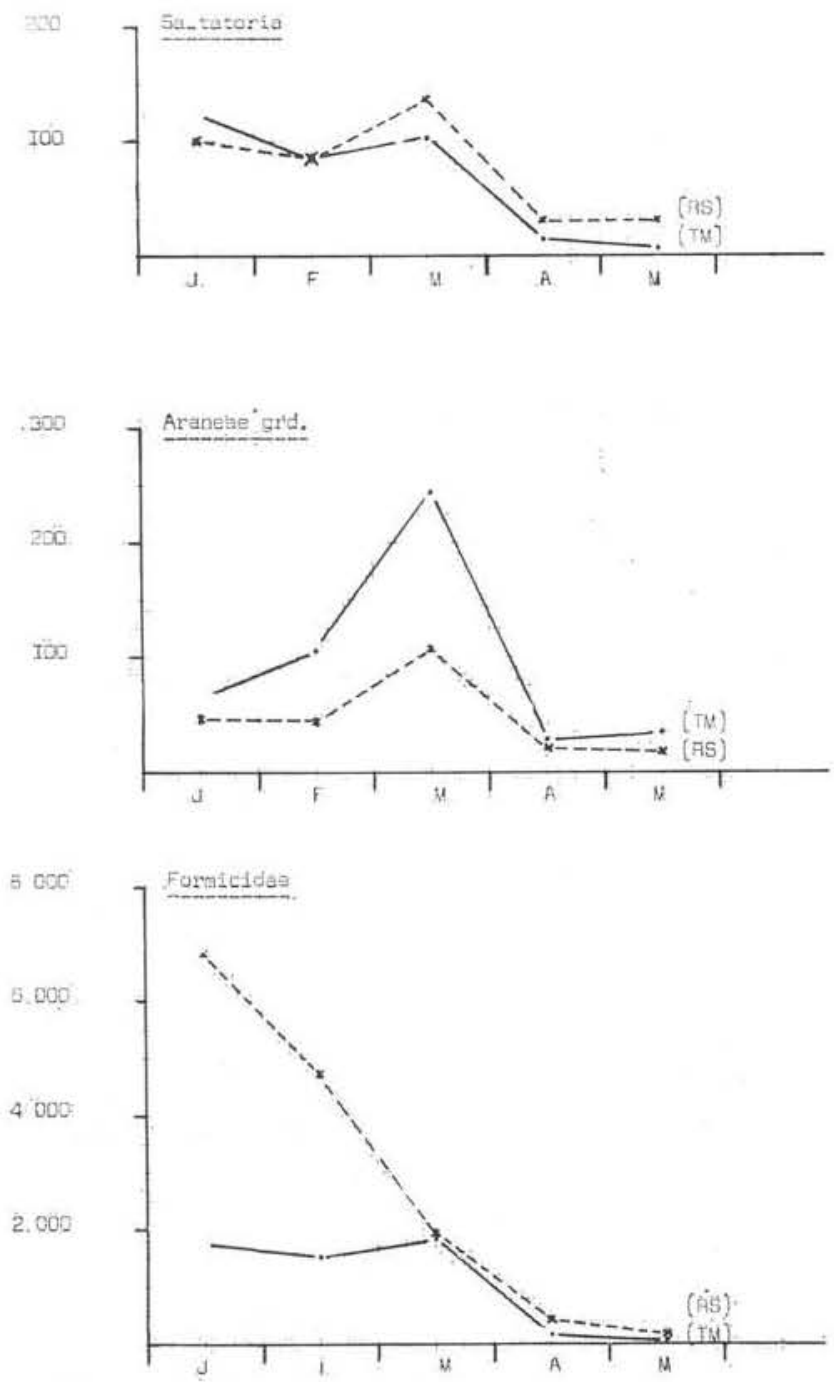

Fig. $2-\mathrm{N}^{\circ}$ de indivíduos coletados por mês (janeiro-maio 1976). Rio Solimões (RS), rio Tarumã-mirim (TM).

As populações da floresta inundável mostram assim três diferentes características :

1. Ascenção no tronco das árvores, da fauna do solo no início da enchente (ex.: Aranhas grandes, rio Solimões / rio Tarumã-mirim; veja fig. 2);

2. Maior densidade de população na área do tronco durante o período de seca; diminuição contínua do número de capturas duran. te a enchente (ex.: Formigas, rio Solimões: veja fig. 2);

3. Densidade de populações estáveis na área do tronco até a inundação da área expe- rimental (março 1976); diminuição abrupta do número de capturas depois da inundação (ex.: Saltatórias, rio Solimões / rio Tart!mã-mirim; veja fig. 2).

IIPOS DE MIGRAÇÃO

A migração dos Artrópodos, para as árvores, foi principalmente verificada no mês de março (ve;a tabela IV, veja fig. 2).

$\mathrm{Na}$ área experimental do rio Tarumã-mirim existem dois tipos de migração:

1. Os grupos de Artrópodos começam a mi. grar já quatro a seis semanas antes da inundação da área (veja tabela VI: tipo I, veja fiy. 3: ex.: Pseudoescorpiōes);


Fig. 3 - Rio Tarumã-mirim, n. ${ }^{\circ}$ de indivíduos coletados por mês (janeiro-maio 1976). 
TABELA V - Artrópodos capturados por meio de fotoeclectores de árvore: Rio Solimões/Rio Tarumã-mirim (janeiro-maio 1976)

A migrantes

RS

Araneae grd.

TM

Araneae grd.

Pseudoscorp.

Araneae peq.

Isopoda

Chilopoda

Symphyla

Blattidae lv.

Uropygi

B população diminui até maio

RS

Formicoidea

Blattidae lv.

Homoptera lv.

Mantidae

Hemiptera

TM

Coleoptera

Lepidoptera Iv.
C população estável até março, depois diminui

RS

TM

Saltatoria

Saltatoria

Formicoidea

Hymenoptera

$\Lambda$ pidae

Mantidae

Machilidae

Hemiptera
2. Os grupos de Artrópodos começam as migraçōes só pouco antes da inundação da área (veja tabela $\mathrm{VI}$ : tipo II, veja fig. 3: ex.: Aranhas grandes).

Além disso foi observado que dos Pseudoescorpiōes, as pequenas espécies e ninfas jovens começam primeiro a migrar (veja tabela IV) .

As espécies e ninfas maiores foram capturadas pela primeira vez cerca de duas a três semanas antes da inundação da área.

TABELA VI (") - Artrópodos capturados por meio de fotoeclectores de árvore: "migrações dos insetos" - Rio Tarumã-mirim - (março 1976)

\begin{tabular}{|c|c|c|c|c|c|c|}
\hline \multirow[b]{2}{*}{ tipo } & & \multicolumn{5}{|c|}{$\begin{array}{l}\mathrm{n} \cdot{ }^{\circ} \text { de artrópodos coletados } \\
\text { por semana }(1 .-31.3 .1976)\end{array}$} \\
\hline & & I & II & III & IV & V \\
\hline I & Pseudoscorp. & 577 & 1008 & 976 & 1360 & 1166 \\
\hline I & Chilopoda & 80 & 76 & 128 & 156 & 214 \\
\hline I & Symphyla & 97 & 169 & 129 & 174 & 117 \\
\hline I & Araneae grd. & 23 & 63 & 31 & 43 & 84 \\
\hline II & Araneae peq. & 76 & 94 & 161 & 263 & 4260 \\
\hline II & Isopoda & 4 & 2 & 3 & 3 & 1390 \\
\hline II & Diplopoda & 13 & 15 & 26 & 19 & 121 \\
\hline II & Uropygi & I & 4 & I & 1 & 43 \\
\hline
\end{tabular}

(*) - Dados preliminares.

\section{Discussão}

Até agora os dados de capturas representam tendências, que só podem ser afirmadas depois de uma avaliação das capturas dos fotoeclectores de solo e das armadilhas de solo. Afirmaçōes finais sobre a fenologia e abundância dos grupos de Artrópodos, só podem ser feitas depois de um ciclo anual. A diferença no inventálio das espécies nas duas áreas experimentais, depende com grande probabilidade da ausência da densa e grossa camada de folhas sobre o solo nas florestas das margens do rio Solimões (compare Irmler, 1974). Além das Formigas, que eram dominantes, foram encontrados nesta área em grande quantidade Artrópodos ou larvas de Artrópodos voadores. Um número pequeno de indivíduos da fauna típica do solo (Dermápteros, Diplópodos, Quilópodos e Pseudoescorpiões) foi encontrado durante a coleta manual direta sob cascas de árvores mortas e caídas.

As Colêmbolas que não foram consideradas durante a avaliação até o momento, são (depois de feito um cáiculo cuidadoso) duas até três vezes mais freqüentes do que as Formigas, na área experimental do rio Solimões e duas até três vezes mais freqüente na área experimental do rio Tarumã-mirim do que na do rio Solimões. Deve ser examinado se as Colêmbolas representam o principal alimento das Formigas e se assim pode ser explicado o baixo número de Colêmbolas do rio Solimões e o maior número de Formigas. A utilização de um novo tipo de fotoeclector de árvore, deve fornecer esclarecimento sobre a descida no tronco dos grupos de Artrópodos no fim da fase de enchente. Investigações sobre a velocidade de colonização do solo vão ser realizadas principalmente na área experimental do rio Tarumãmirim. Dentre outras investigações, deveria ser verificado o espectro de alimentação e principalmente o biótopo dos grupos e espécies de Artrópodos. 
Para melhor se entender o funcionamento do ecossistema "Floresta Inundável", são absolutamente necessários trabalhos contínuos, sobre, por exemplo, a determinação da biomassa, produtividade e consumo de energia das espécies dominantes de Artrópodos.

\section{Agradecimentos}

A Fundação de Estudos do Povo Alemão (Studienstiftung des Deutschen Volkes), pela oportunidade de realizar a pesquisa; ao Instituto Nacional de Pesquisas da Amazônia (INPA), pelo apoio prestado em Manaus (Projeto OEA), bem como ao Conselho de Pesquisas Alemão (Deutsche Forschungsgemeinschaft), através do Projeto Prof. Dr. W. Funke.

\section{Summary}

Experiments have been conducted in a black-water region (rio Tarumâ-mirim) an in a white-water region (rio Solimōes) since January 1976. Each experimental area contained 28 pitfall-traps, 4 ground photo-eclectors and 13 arboreal photoeclectors (Minimal program for an ecosystem-analysis: Grimm, Funke \& Schauermann, 1974). In the Solimões area, the arboreal photo-eclectors have collected 18.197 arthropods and Anura up to May 1976. Formicoidea $(14.068=77,31 \%)$ and Diptera $(1.634=8,98 \%)$ have been dominant. In the rio Tarumã-mirim area 27.594 arthropods and Gastropoda have been collected during the same period. Pseudoscorpiones $(7.942=28,28 \%)$, small Araneae $(5.878=31,30 \%)$ and Formicoidea $(5.422=19,65 \%)$ have been dominant. A lack of soil-fauna is found in the Solimões area, for there is no ground-litter, and only the big Araneae migrate before the rising water. The rio Tarumã-mirim area contained 8 groups of arthropods, particularly the soil-fauna, which migrate. Some arthropods migrate 6-8 weeks before the area is flooded, others wait until the last week prior to inundation.

\section{BIBLIOGRAFIA CITADA}

ADIS, J.

1974 - Bodenfallenfaenge in einem Buchenwald und ihr Aussagewert. Diplomarbeit an der Naturwissenschaftlichen Fakultaet der Universitaet Göttingen.

AdIs, J. \& KRAMER, E.

1975 - Formaldehyd-Loesung attrahiert Carabus problematicus (Coleoptera: Carabidae). - Entomol. Germ., 2(2): 121-125; Stuttgart.
BECK, L.

1968 - Zum Jahreszeitlichen Massenwechsel zweier Oribatidenarten (Acari) im neotropischen Ueberschwemmungswald. Verh, dtsch. Zool. Ges, : 535-540; Innsbruck.

1971 - Bodenzoologische Gliederung und Charakterisierung des amazonischen Regenwaldes, Amazoniana, 3:69-132; Kiel.

1972 - Der Einfluss der jahresperiodischen Ueberflutungen auf den Massenwechsel der Bodenarthropoden im zentral-amazonischen Regenwaldgebiet. Pedobiologia, 2 : 133-148.

BECK, L. \& SCHUBART, H.

1968 - Zur Coleopterenfauna amazonischer Boeden Amazoniana, 1:311-322; Kiel.

Ellenberg, $\mathrm{H}$.

1971 - Integrated Experimental Ecology. Ecol. Studies, 2:1-15; Berlin.

FUNKE, W.

1971 - Food and Energy Turnover of Leafeating Insects and their Influence on Primary Production. Ecol. Studies, 2 : 81-93; Berlin.

Grimm, R.; Funke, W. \& Schauermann, J.

1974 - Minimalprogramm zur Oekosystemanalyse: Untersuchungen an Tierpopulationen in Wald-Oekosystemen. Verh. Ges. Oekol.: 77-87; Erlangen.

IRMLER, U

1973 - Population-Dynamic and physiological Adaption of Pentatomia egregia Chaud. (Col. Cicindelidae) to the Amazonian Inundation Forest. Amazoniana, 4: 219-227; Kiel.

1974 - Vergleichende oekologische Studien an der aquatischen Makrobenthosfauna zentralamazonischer Ueberschwemmungswaelder. - Dissertation an der Mathematisch - Naturwissenschaftlichen Fakultaet der Universitaet Kiel.

JANZEN, D.H.

1974 - Tropical Blackwater Rivers, Animals and Mast Nruiting by the Dipterocarpaceae. Biotropica, 6(2) : 69-103.

SCHALlER, F.

1969 - Zur Frage des Formensehens bei Collembolen. Verh. dtsch. Zool. Ges, : 368-375.

1973 - Zur Ernaehrungsbiologie neotropischer Bodentiere. - Naturwissenschaften, 4: 203.

Sioli, H.

1956 - Ueber Natur und Mensch im bras1lianischen Amazonasgebiet. Erdkunde, 10 : 89-109; Bonn. 